Original article

\title{
Sarcopenic obesity and overall mortality: Results from the application of novel models of body composition phenotypes to the National Health and Nutrition Examination Survey 1999-2004
}

\author{
Carla Van Aller ${ }^{\text {a }}$, Jose Lara ${ }^{\text {b }}$, Blossom C.M. Stephan ${ }^{\text {c }}$, Lorenzo Maria Donini ${ }^{\mathrm{d}}$, \\ Steven Heymsfield ${ }^{\mathrm{e}}$, Peter T. Katzmarzyk ${ }^{\mathrm{e}}$, Jonathan C.K. Wells ${ }^{\mathrm{f}}$, Carla M. Prado ${ }^{\mathrm{g}}$, \\ Mario Siervo ${ }^{\text {a, * }}$ \\ ${ }^{a}$ Human Nutrition Research Centre, Institute of Cellular Medicine, Newcastle University, Campus for Ageing and Vitality, Newcastle on Tyne, NE4 5PL, UK \\ b Department of Applied Sciences, Faculty of Health and Life Sciences, Northumbria University, UK \\ ' Institute of Health and Society, Newcastle University, Campus for Ageing and Vitality, Newcastle on Tyne, NE4 5PL, UK \\ d Department of Experimental Medicine- Medical Pathophysiology, Food Science and Endocrinology Section, Sapienza University of Rome, 00185 Rome, Italy \\ e Pennington Biomedical Research Center, Baton Rouge, LA, USA \\ ${ }^{\mathrm{f}}$ Childhood Nutrition Research Centre, Institute of Child Health, University College of London, 30 Guilford Street, WC1N 1EH, London, UK \\ ${ }^{g}$ Department of Agriculture, Food and Nutritional Science, University of Alberta, Edmonton, AB, Canada
}

\section{A R T I C L E I N F O}

\section{Article history:}

Received 25 July 2017

Accepted 12 January 2018

\section{Keywords:}

Sarcopenic obesity

Body composition

Mortality

\begin{abstract}
S U M M A R Y
Background/Objectives: There is no consensus on the definition of sarcopenic obesity (SO), resulting in inconsistent associations of SO with mortality risk. We aim to evaluate association of dual energy x-ray absorptiometry (DXA) SO models with mortality risk in a US adult population ( $\geq 50$ years).

Subjects/Methods: The study population consisted of 3577 participants aged 50 years and older from the 1999-2004 National Health and Nutrition and Examination Survey with mortality follow-up data through December 31, 2011. Difference in survival time in people with and without So defined by three body composition DXA models (Model 1: body composition phenotype model; Model 2: Truncal Fat Mass (TrFM)/Appendicular Skeletal Muscle Mass (ASM) ratio model; Model 3: Fat Mass (FM)/Fat Free Mass (FFM) ratio). The differences between the models were assessed by the acceleration failure time model, and expressed as time ratios (TR).

Results: Participants age 50-70 years with SO had a significantly decreased survival time, according to the body composition phenotype model (TR: 0.92; 95\% CI: 0.87-0.97), and TrFM/ASM ratio model (TR: 0.88; 95\% CI: 0.81-0.95). The FM/FFM ratio model did not detect significant differences in survival time. Participants with SO aged 70 years and older did not have a significantly decreased survival time, according to all three models.

Conclusions: A SO phenotype increases mortality risk in people of age 50-70 years, but not in people aged 70 years and older. The application of the body composition phenotype and the TrFM/ASM ratio models may represent useful diagnostic approaches to improve the prediction of disease and mortality risk.
\end{abstract}

() 2018 Elsevier Ltd and European Society for Clinical Nutrition and Metabolism. All rights reserved.

\section{Introduction}

Body composition is influenced by different physiological and non-physiological factors such as ageing, gender, diet and physical

\footnotetext{
The material presented in this manuscript is original and it has not been sub mitted for publication elsewhere while under consideration in Clinical Nutrition.

* Corresponding author.

E-mail address: mario.siervo@ncl.ac.uk (M. Siervo).
}

activity, or acute and chronic illnesses [1]. These factors contribute to shape the overall distribution of population body composition phenotypes as they are key risk factors, amongst others, for increased adiposity and loss of lean body mass [2-4] and ageing may represent an important modifier of these reciprocal body composition changes [5]. The net result of these biological, lifestyle and demographic trends could be an increase in the prevalence of the sarcopenic obesity (SO) phenotype, defined as the co-occurrence of high adiposity and low lean body mass in the same individual [6]. 
Body Mass Index (BMI) is the most widely used indicator to assess adiposity. However, a limitation of BMI is its inability to distinguish the proportion of fat and lean body mass [7]. This limitation can be overcome using different body composition methods and dual energy x-ray absorptiometry (DXA) may offer the best compromise to cost, accuracy and reproducibility [8].

DXA is currently considered as one of the most accurate body composition methods for the assessment of SO [9]. However, there is no consensus as yet on the definitions of SO [9-11]. Consequently, the application of different definitions of SO has been an important limiting factor in trying to establish its predictive role for disease risk and mortality. Important drawbacks of many SO definitions are the lack of control for between-subject differences in body mass, use of young populations as reference groups and assessment of adiposity using anthropometric indexes (i.e., BMI, waist circumference). The consequences of these differences are the inconsistent association of SO with mortality in studies reporting significant [12-18] and non-significant associations $[19,20]$.

Novel DXA models for the assessment of SO and other body composition phenotypes have been proposed, which allow for the control of the confounding effects of age, sex and BMI [21,22]. These models were developed from DXA data of the U.S. National Health and Nutrition Examination Survey (NHANES) 1999-2004. The aims of these analyses are to verify if these newly proposed DXA models are significant predictors of increased or decreased survival time in a US adult population (age 50 years and older), if these models predict survival time better than body mass index (BMI), and, finally, to obtain more insights into the association between $\mathrm{SO}$ and mortality risk.

\section{Methods}

\subsection{NHANES}

Data were obtained from the NHANES 1999-2004. The NHANES is a survey of the non-institutionalized civilian resident population of the United States. A complex, multistage probability sampling design was used to select a representative sample of 14,200 participants [23]. The NHANES data on mortality for public use are available continuously for the entire 1999-2004 period. Mortality data were available from the date of survey participation to December 31, 2011 [24] and the follow-up period ranged between 7 and 12 years. Mortality status is determined by conducting a probabilistic pairing between NHANES and records of death certificates from the National Death Index. Detailed information about NHANES and the mortality data can also be found elsewhere $[23,25]$. For this analysis, participants weighing more than $136 \mathrm{~kg}$, taller than $1.96 \mathrm{~m}(\mathrm{n}=3707)$, below 50 years of age $(\mathrm{n}=6306)$, who died in less than 24 months after the baseline survey $(n=121)$, and with missing data for household income level $(n=470)$, average daily physical activity level $(n=10)$, education level $(n=9)$, and mortality status ( $\mathrm{n}=7$; total participants with missing data: $\mathrm{n}=489$ ) were excluded from the analysis. The final sample consisted of 3577 participants.

\section{2. $D X A$}

Body composition assessment was undertaken by DXA (Hologic QDR 4500A) [24]. Participants were not eligible for a DXA scan if they were pregnant, weighed more than $136 \mathrm{~kg}$ and if they were taller than $1.96 \mathrm{~m}$. In addition, participants were not eligible if they had been exposed to radiographic contrast material in the past 7 days or nuclear medicine in the past 3 days [24]. Complete DXA data were obtained from $80 \%$ of the eligible participants [25]. DXA data incompleteness was related to age, BMI, weight and height, and multiple imputation of the missing data was performed by the National Center for Health Statistics (NCHS). Five completed data files containing both the non-missing and imputed DXA data values were created [25].

\subsection{BMI and DXA-models of body composition phenotypes}

Nutritional status and body composition were defined to analyse if subgroups based on these variables have an increased or decreased survival time. Nutritional status was defined by BMI (body weight/height ${ }^{2}$ ), and categorized for analysis as BMI $<25.0 \mathrm{~kg} / \mathrm{m}^{2}, 25.0-30.0 \mathrm{~kg} / \mathrm{m}^{2}$ and $\geq 30.0 \mathrm{~kg} / \mathrm{m}^{2}$. Body composition and SO were assessed by three different models based on DXA data $[21,22]$. These three approaches have in common that they divide people into groups based on specific cut-points of muscle and fat mass. The cut-points are defined from age-standardised reference curves stratified by BMI and gender. The reference curves were developed from non-imputed NHANES 1999-2004 DXA data of 13,236 participants above 18 years. The first approach, FM/FFM model, was based on the ratio between total fat mass (FM) and total fat free mass (FFM) [22]. The cut-off points of the reference curves for this approach were as follows: a ratio below the 15th centile, in the 15 th -85 th centile, in the 85 th -95 th centile or above the 95th centile. The group with a FM/FFM ratio above the 95th centile are considered as the SO group. The second approach, BC phenotype model, divides people in four different body composition phenotypes based on having low adiposity (LA) or high adiposity (HA) and low muscle mass (LM) or high muscle mass (HM) [21]. Participants were defined as having low or high adiposity when the fat mass index (total fat mass/height ${ }^{2}$ ) was below or above the 50th percentile of the reference curve. The same applies for low or high muscle mass, but then with reference curves of the appendicular skeletal muscle index (lean soft tissue of the arms and legs/ height ${ }^{2}$ ). The group with HA and LM are considered as the SO group. The third, and most specific approach at the regional anatomic level, is the TrFM/ASM model. This approach was based on the ratio between truncal fat mass (TrFM) and appendicular skeletal muscle mass (ASM) [22], and similar to the FM/FFM model approach. The only difference was in the body components used for the calculation of the ratio. Assessment of body composition, according to the approaches mentioned above, were performed with an automated toolkit which can be made available upon request to the corresponding author (MS). Detailed information about the body composition models has been published previously [21,22].

\subsection{Covariates}

All covariates were self-reported. Ethnicity was classified as non-Hispanic white, Mexican American, non-Hispanic black or other ethnicity. Education level is the highest grade or level of school completed or the highest degree received, and classified as an education level lower than high school, high school, or higher than high school. The smoking status of participants was divided in smokers and non-smokers. Participants were classified as smoker if they regularly (some days or every day) smoked cigarettes, cigars or pipe or if they chewed tobacco. Average daily physical activity was classified as participants sits a lot; stands or walks a lot; lift light loads or climbs stairs or hills often; or does heavy work or carries heavy loads.

\subsection{Statistical analysis}

Descriptive statistics are expressed as mean \pm standard error (continuous variables) or as percentages (categorical variables). 
Accelerated Failure Time (AFT) models were used to determine the association between time-to-event (e.g. mortality) and: (1) BMI; and, (2) the three body composition models. The AFT models are parametric models that assume a specific distribution, but do not require the assumption of proportional hazards (i.e. for Cox regression, which was not upheld in the data) [26]. Five different AFT models were fit assuming different distributions for the baseline hazard function (i.e. Weibull, exponential, Gamma, log-logistic and log-normal) and model performance compared fit using the Akaike Information Criterion (AIC) and Bayesian Information Criterion (BIC). A final model assuming the log-normal distribution best fitted the data for the BMI and each of the three DXA-models of body composition phenotypes. If stratified analysis were performed the log-logistic distribution fitted best for participants aged 50-70 years, and the exponential distribution for participants aged 70 years and older. Outcomes are expressed as time ratios (TR) with $95 \%$ confidence intervals $(95 \% \mathrm{CI})$. Age at death or the year 2011 was used as the time-scale with age at baseline survey used as the entry time [27]. Both uni-variable and multivariable models adjusting for sex, ethnicity, education level, household income level, smoking status and average daily physical activity were run to test the association between body composition, defined by each model, with survival time. For each model, the reference group included those participants with a body composition that was assumed healthiest, i.e. low fat mass and high muscle mass. All statistical analyses were performed using the survey procedure of STATA version 14.2, which accounts for the complex sample design and DXA multiple imputation procedure of the NHANES dataset.

\section{Results}

Baseline characteristics of the sample are shown in Table 1. The total study population consisted of 3577 participants of 50 years and older, of which $15.4 \%$ were deceased in 2011. The subgroup of participants aged 50-69 years consisted of 2424 participants, of which $8.4 \%$ were deceased in 2011 . The subgroup of participants of 70 years and older consisted of 1153 participants, of which $39.4 \%$ were deceased in 2011. The baseline characteristics of the 610 participants excluded from the analyses, because of missing data or deceased within 24 months after the baseline survey, are shown in Table 1 of the Online Supplementary Material. Overall, participants had similar characteristics for age, BMI, socio-demographic factors and prevalence of body composition phenotypes to the population included in the main analysis.

The association between BMI category and survival years is shown in Table 2. There was no significant association between BMI and survival years for all participants. The same was observed for the subgroup analysis of the age groups $50-70$ years and 70 years and older.

In Table 3 the association between the FM/FFM model and survival is shown. There is no significant association for participants 50 years and older with a certain FM/FFM ratio and survival years. The same was observed for the subgroup analysis of the participants 50-70 years. In the subgroup analysis of the participants 70 years and older the participants with a FM/FFM ratio in the 15th-85th centile (TR: 1.53; 95\% CI: 1.01-2.33) had a significant longer survival in comparison with the reference group.

The association between $\mathrm{BC}$ phenotype model and survival years is shown in Table 4. Participants 50 years and older with LALM (TR: 0.95; 95\% CI: 0.92-0.99) and HA-LM (SO, TR: 0.96; 95\% CI: 0.92-0.99) had a significantly shorter survival compared to the participants with a LA-HM. The association became stronger in the subgroup analysis for participants aged 50-70 years with LA-LM (TR: $0.93 ; 95 \% \mathrm{CI}: 0.88-0.98$ ) and HA-LM (SO, TR: 0.92; 95\% CI: 0.87-0.97). In addition, participants with a HA-HM had a
Table 1

Baseline characteristics of a representative US adult population of 50 years and older.

\begin{tabular}{|c|c|c|c|}
\hline & $\begin{array}{l}\text { Participants } \\
50+\text { years }\end{array}$ & $\begin{array}{l}\text { Participants } \\
50-70 \text { years }\end{array}$ & $\begin{array}{l}\text { Participants } \\
70+\text { years }\end{array}$ \\
\hline Participants, $\mathrm{n}^{\mathrm{a}}$ & 3577 & 2424 & 1153 \\
\hline Men, \% & 48.6 & 50.7 & 41.3 \\
\hline Age at survey, mean (SE) & $61.8(0.2)$ & $57.6(0.2)$ & $76.2(0.1)$ \\
\hline Decedents, \% & 15.4 & 8.4 & 39.4 \\
\hline \multicolumn{4}{|l|}{ Ethnicity } \\
\hline Non-Hispanic white, \% & 79.1 & 77.7 & 84.1 \\
\hline Mexican American, \% & 3.9 & 4.2 & 2.9 \\
\hline Non-Hispanic black, \% & 8.2 & 8.7 & 6.7 \\
\hline Ethnicity other, \% & 8.8 & 9.5 & 6.3 \\
\hline \multicolumn{4}{|l|}{ Education level } \\
\hline$<$ High school, \% & 22.3 & 19.1 & 33.1 \\
\hline High school, \% & 26.3 & 25.0 & 30.7 \\
\hline >High school, \% & 51.4 & 55.9 & 36.2 \\
\hline \multicolumn{4}{|l|}{ Household income level } \\
\hline$<\$ 20.000, \%$ & 20.1 & 16.1 & 33.9 \\
\hline$\$ 20.000-\$ 65.000, \%$ & 49.0 & 48.0 & 52.3 \\
\hline$>\$ 65.000, \%$ & 30.9 & 35.9 & 13.8 \\
\hline Smoker, \% & 19.6 & 22.6 & 9.3 \\
\hline \multicolumn{4}{|l|}{$\begin{array}{l}\text { Average daily } \\
\text { physical activity }\end{array}$} \\
\hline Sits a lot during the day, \% & 25.8 & 5.3 & 27.5 \\
\hline $\begin{array}{l}\text { Stands or walks a } \\
\text { lot during the day, \% }\end{array}$ & 55.1 & 54.2 & 58.4 \\
\hline Lifts light loads during the day, \% & 14.8 & 15.4 & 12.7 \\
\hline $\begin{array}{l}\text { Does heavy work or } \\
\text { carries heavy loads, \% }\end{array}$ & 4.3 & 5.1 & 1.4 \\
\hline \multicolumn{4}{|l|}{ BMI } \\
\hline$<18.5 \mathrm{~kg} / \mathrm{m}^{2}, \%$ & 0.2 & 0.1 & 0.6 \\
\hline $18.5-25.0 \mathrm{~kg} / \mathrm{m}^{2}, \%$ & 26.8 & 25.6 & 30.6 \\
\hline $25.0-30.0 \mathrm{~kg} / \mathrm{m}^{2}, \%$ & 40.1 & 39.0 & 44.0 \\
\hline$\geq 30.0 \mathrm{~kg} / \mathrm{m}^{2}, \%$ & 32.9 & 35.3 & 24.8 \\
\hline \multicolumn{4}{|l|}{ Body composition phenotypes ${ }^{\mathrm{b}, \mathrm{c}}$} \\
\hline LA-HM, \% & 23.4 & 23.2 & 23.9 \\
\hline LA-LM, \% & 25.7 & 26.1 & 24.4 \\
\hline HA-HM, \% & 26.9 & 27.4 & 25.3 \\
\hline HA-LM, \% & 24.0 & 23.4 & 26.4 \\
\hline \multicolumn{4}{|l|}{ TrFM/ASM centiles ${ }^{\mathrm{b}, \mathrm{d}}$} \\
\hline$<15$ th centile, $\%$ & 12.4 & 12.0 & 13.9 \\
\hline $15-85$ th centile, $\%$ & 71.9 & 72.4 & 70.3 \\
\hline $85-95$ th centile, $\%$ & 9.9 & 9.9 & 9.9 \\
\hline$\geq 95$ centile, $\%$ & 5.8 & 5.7 & 5.9 \\
\hline \multicolumn{4}{|l|}{ FM/FFM centiles ${ }^{\mathrm{b}, \mathrm{d}}$} \\
\hline$<15$ th centile, $\%$ & 13.2 & 13.0 & 14.4 \\
\hline $15-85$ th centile, $\%$ & 70.6 & 71.2 & 68.7 \\
\hline $85-95$ th centile, $\%$ & 10.4 & 10.1 & 11.4 \\
\hline$\geq 95$ centile, $\%$ & 5.7 & 5.6 & 5.5 \\
\hline
\end{tabular}

Complex survey design is taken into account for calculating the baseline characteristics, unless stated otherwise.

a Real observations, complex survey design is not taken into account.

b Multiple imputed data.

${ }^{c}$ LA is low adiposity, HA is high adiposity, LM is low adiposity and HM is high muscle mass.

${ }^{d}$ TrFM is truncal fat mass, ASM is appendicular skeletal muscle mass, FM is fat mass and FFM is fat free mass. The groups are formed based on specific body composition ratio reference curves.

significant shorter survival compared to the participants with LAHM (TR: 0.95; 95\% CI: 0.90-1.00). In the subgroup analysis for the participants aged 70 years and older the direction of the association changed. The participants with HA-HM (TR: 1.42 ; $95 \% \mathrm{CI}$ : 1.07-1.89) survived significantly longer compared to the group with LA-HM.

The association between TrFM/ASM model and survival years is shown in Table 5. There is no significant association for participants 50 years and older with TrFM/ASM ratio and survival years when adjusted for confounders. In the subgroup analysis the participants 50-70 years with a TrFM/ASM ratio in the 85th-95th centile (TR: $0.91 ; 95 \% \mathrm{CI}: 0.84-0.99$ ) and above the 95th centile (SO, TR: 0.88; $95 \% \mathrm{CI}: 0.81-0.95$ ) had a significantly shorter survival compared to 
Table 2

Time ratios (TR) for the association between BMI and survival years.

\begin{tabular}{|c|c|c|c|c|c|c|c|c|c|}
\hline & \multicolumn{3}{|c|}{ Participants $\geq 50$ years ${ }^{a}$} & \multicolumn{3}{|c|}{ Participants $50-70$ years $^{\mathrm{b}}$} & \multicolumn{3}{|c|}{ Participants $\geq 70$ years $^{c}$} \\
\hline & TR & $95 \% \mathrm{CI}$ & p-value & TR & $95 \% \mathrm{CI}$ & p-value & TR & $95 \% \mathrm{CI}$ & p-value \\
\hline \multicolumn{10}{|l|}{ Crude model } \\
\hline $\mathrm{BMI}<25 \mathrm{~kg} / \mathrm{m}^{2}$ & 1.00 & & & 1.00 & & & 1.00 & & \\
\hline BMI $25-30 \mathrm{~kg} / \mathrm{m}^{2}$ & 1.03 & $1.00-1.06$ & 0.06 & 1.04 & $0.99-1.08$ & 0.10 & 1.01 & $0.99-1.03$ & 0.48 \\
\hline $\mathrm{BMI} \geq 30 \mathrm{~kg} / \mathrm{m}^{2}$ & 0.99 & $0.96-1.02$ & 0.61 & 0.99 & $0.94-1.03$ & 0.50 & 0.99 & $0.97-1.01$ & 0.47 \\
\hline \multicolumn{10}{|l|}{ Adjusted model $^{\mathrm{d}}$} \\
\hline $\mathrm{BMI}<25 \mathrm{~kg} / \mathrm{m}^{2}$ & 1.00 & & & 1.00 & & & 1.00 & & \\
\hline BMI $25-30 \mathrm{~kg} / \mathrm{m}^{2}$ & 1.03 & $1.00-1.07$ & 0.06 & 1.04 & $0.99-1.08$ & 0.09 & 1.20 & $0.95-1.53$ & 0.13 \\
\hline $\mathrm{BMI} \geq 30 \mathrm{~kg} / \mathrm{m}^{2}$ & 0.99 & $0.96-1.03$ & 0.65 & 0.99 & $0.94-1.04$ & 0.62 & 1.22 & $0.92-1.61$ & 0.16 \\
\hline
\end{tabular}

BMI is body mass index. Complex survey design is taken into account for calculating the TR.

a Accelerated failure time model analysis with lognormal distribution.

b Accelerated failure time model analysis with loglogistic distribution.

c Accelerated failure time model analysis with exponential distribution.

d Adjusted for sex, ethnicity, education level, household income level, smoking status and average daily physical activity.

Table 3

Time ratios (TR) for the association between FM/FFM ratio and survival years.

\begin{tabular}{|c|c|c|c|c|c|c|c|c|c|}
\hline & \multicolumn{3}{|c|}{ Participants $\geq 50$ years $^{\mathrm{a}}$} & \multicolumn{3}{|c|}{ Participants $50-70$ years ${ }^{b}$} & \multicolumn{3}{|c|}{ Participants $\geq 70$ years $^{c}$} \\
\hline & TR & $95 \% \mathrm{CI}$ & p-value & TR & $95 \% \mathrm{CI}$ & p-value & TR & $95 \% \mathrm{CI}$ & p-value \\
\hline \multicolumn{10}{|l|}{ Crude model } \\
\hline$<15$ th centile & 1.00 & & & 1.00 & & & 1.00 & & \\
\hline 15-84th centile & 1.02 & $0.98-1.06$ & 0.31 & 1.01 & $0.96-1.07$ & 0.68 & 1.20 & $0.91-1.59$ & 0.18 \\
\hline 85-94th centile & 1.01 & $0.96-1.06$ & 0.70 & 0.98 & $0.92-1.05$ & 0.58 & 1.44 & $0.94-2.19$ & 0.09 \\
\hline$>95$ th centile & 0.99 & $0.93-1.05$ & 0.66 & 0.96 & $0.90-1.03$ & 0.24 & 1.12 & $0.71-1.76$ & 0.62 \\
\hline \multicolumn{10}{|l|}{ Adjusted model $^{\mathrm{d}}$} \\
\hline$<15$ th centile & 1.00 & & & 1.00 & & & 1.00 & & \\
\hline 15-84th centile & 1.01 & $0.97-1.06$ & 0.54 & 0.99 & $0.93-1.05$ & 0.81 & 1.28 & $0.96-1.71$ & 0.09 \\
\hline 85-94th centile & 1.00 & $0.95-1.05$ & 0.98 & 0.96 & $0.90-1.03$ & 0.24 & 1.53 & $1.01-2.33$ & 0.04 \\
\hline$>95$ th centile & 1.00 & $0.94-1.06$ & 0.96 & 0.96 & $0.89-1.04$ & 0.35 & 1.31 & $0.82-2.09$ & 0.26 \\
\hline
\end{tabular}

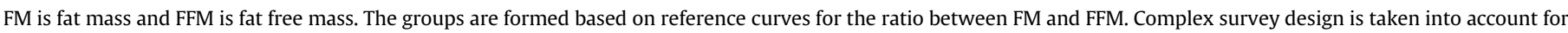
calculating the TR.

a Accelerated failure time model analysis with lognormal distribution.

b Accelerated failure time model analysis with loglogistic distribution.

c Accelerated failure time model analysis with exponential distribution.

d Adjusted for sex, ethnicity, education level, household income level, smoking status and average daily physical activity.

the participants with a TrFM/ASM ratio below the 15th centile. In the subgroup analysis of the participants 70 years and older the direction of the association changed. Participants with a TrFM/ASM ratio in the 15 th-85th (TR: 1.43 ; $95 \% \mathrm{CI}$ : $1.14-1.79$ ) had a significantly longer survival in comparison with the reference group.

\section{Discussion}

This study showed that BMI did not predict increased or decreased survival time in adults of 50 years and older. We showed for the first time that the DXA based BC phenotype and TrFM/ASM models significantly predicted survival time. However, the association was age-dependent as the participants with SO was associated with lower survival time in participants of 50-70 years, but not in participants older than 70 years of age.

Specifically, significant differences in survival time were found between the body composition groups identified by the $\mathrm{BC}$ phenotype and the TrFM/ASM models. Therefore, these two models outperform BMI and the FM/FFM ratio model for the prediction of survival time in this population. The performance of the $\mathrm{BC}$ phenotype model could be explained by the use of ASM cut offs for the identification of sarcopenia and possibly a better discrimination of the body composition classes across the four different body composition phenotypes (i.e., HA-HM, LA-HM, LA-LM, HA-LM). The significant association of the TrFM/ASM model with survival time may be explained by the inclusion of measures of central adiposity and skeletal muscle mass; therefore, it could represent a more informative model based on the stronger link of these two components with the pathogenesis of cardiovascular and metabolic diseases. ASM is a proxy measure of metabolic control, functional performance and physical disability [28-30] and loss of ASM is associated with poorer metabolic control and increased mortality as well as impaired quality of life [31].

We found that the association between SO and survival time were different in two age groups. The SO group aged 50-70 years had the lowest survival if defined by the BC phenotype model and TrFM/ASM ratio model. The SO group aged 70 years and older was instead not associated with a decreased survival time according to both models. The significant association of SO with increased mortality risk in the age group 50-70 years confirmed results found in other studies [12-18].

A recent study used the same NHANES 1999-2004 dataset to evaluate the association between SO and risk of overall mortality [18]. SO was defined by gender specific cut-off points for ASM and FM and adopted a diagnostic approach similar to our BC phenotype model. However, the two analyses were different for the choice of age cut offs ( $\geq 50$ years $v s \geq 60$ years) and stratification of the analyses (age- $v s$ gender-stratified). A mid-life cut off point for age ( $\geq 50$ years) was chosen for our analyses based on the capacity of our models to account for age in the identification of body composition phenotypes and considering mid-life as a critical life stage where ill health becomes the major cause of death, and the number of mortality events progressively increases [32]. In addition, several other studies exploring the association between body composition and health outcomes have used the same age cut off point [16,33-41], and one of them conducted the same age- 
Table 4

Time ratios (TR) of the association between body composition phenotypes and survival years.

\begin{tabular}{|c|c|c|c|c|c|c|c|c|c|}
\hline & \multicolumn{3}{|c|}{ Participants $\geq 50$ years $^{\mathrm{a}}$} & \multicolumn{3}{|c|}{ Participants $50-70$ years $^{\text {b }}$} & \multicolumn{3}{|c|}{ Participants $\geq 70$ years $^{c}$} \\
\hline & TR & $95 \% \mathrm{CI}$ & p-value & TR & $95 \% \mathrm{CI}$ & p-value & TR & $95 \% \mathrm{CI}$ & p-value \\
\hline \multicolumn{10}{|c|}{ Crude model } \\
\hline LA-HM & 1.00 & & & 1.00 & & & 1.00 & & \\
\hline LA-LM & 0.95 & $0.92-0.99$ & 0.008 & 0.94 & $0.89-0.99$ & 0.01 & 0.94 & $0.72-1.23$ & 0.64 \\
\hline HA-HM & 0.99 & $0.96-1.02$ & 0.59 & 0.96 & $0.92-1.01$ & 0.10 & 1.34 & $1.00-1.80$ & 0.04 \\
\hline HA-LM & 0.95 & $0.92-0.99$ & 0.01 & 0.92 & $0.87-0.98$ & 0.007 & 1.05 & $0.78-1.41$ & 0.76 \\
\hline \multicolumn{10}{|c|}{ Adjusted model $^{\mathrm{d}}$} \\
\hline LA-HM & 1.00 & & & 1.00 & & & 1.00 & & \\
\hline LA-LM & 0.95 & $0.92-0.99$ & 0.007 & 0.93 & $0.88-0.98$ & 0.01 & 0.96 & $0.71-1.30$ & 0.79 \\
\hline HA-HM & 0.99 & $0.96-1.02$ & 0.37 & 0.95 & $0.90-1.00$ & 0.03 & 1.42 & $1.07-1.89$ & 0.01 \\
\hline HA-LM & 0.96 & $0.92-0.99$ & 0.01 & 0.92 & $0.87-0.97$ & 0.006 & 1.12 & $0.81-1.54$ & 0.47 \\
\hline
\end{tabular}

LA is low adiposity, HA is high adiposity, LM is low adiposity and HM is high muscle mass. Complex survey design is taken into account for calculating the TR.

a Accelerated failure time model analysis with lognormal distribution.

b Accelerated failure time model analysis with loglogistic distribution.

c Accelerated failure time model analysis with exponential distribution.

d Adjusted for sex, ethnicity, education level, household income level, smoking status and average daily physical activity.

Table 5

Time ratios (TR) for the association between TrFM/ASM ratio and survival years.

\begin{tabular}{|c|c|c|c|c|c|c|c|c|c|}
\hline & \multicolumn{3}{|c|}{ Participants $\geq 50$ years $^{\mathrm{a}}$} & \multicolumn{3}{|c|}{ Participants $50-70$ years $^{b}$} & \multicolumn{3}{|c|}{ Participants $\geq 70$ years $^{c}$} \\
\hline & TR & $95 \% \mathrm{CI}$ & p-value & TR & $95 \% \mathrm{CI}$ & p-value & TR & $95 \% \mathrm{CI}$ & p-value \\
\hline \multicolumn{10}{|l|}{ Crude model } \\
\hline$<15$ th centile & 1.00 & & & 1.00 & & & 1.00 & & \\
\hline 15-84th centile & 1.01 & $0.97-1.04$ & 0.65 & 0.98 & $0.93-1.03$ & 0.47 & 1.34 & $1.07-1.67$ & 0.01 \\
\hline 85-94th centile & 0.98 & $0.92-1.04$ & 0.41 & 0.93 & $0.86-1.00$ & 0.06 & 1.38 & $0.89-2.14$ & 0.15 \\
\hline$>95$ th centile & 0.94 & $0.88-1.00$ & 0.05 & 0.89 & $0.82-0.95$ & 0.002 & 1.41 & $0.85-2.32$ & 0.17 \\
\hline \multicolumn{10}{|l|}{ Adjusted model $^{\mathrm{d}}$} \\
\hline$<15$ th centile & 1.00 & & & 1.00 & & & 1.00 & & \\
\hline 15-84th centile & 1.00 & $0.96-1.04$ & 0.96 & 0.95 & $0.89-1.01$ & 0.10 & 1.43 & $1.14-1.79$ & 0.003 \\
\hline 85-94th centile & 0.98 & $0.92-1.04$ & 0.43 & 0.91 & $0.84-0.99$ & 0.03 & 1.44 & $0.91-2.28$ & 0.11 \\
\hline$>95$ th centile & 0.95 & $0.89-1.01$ & 0.09 & 0.88 & $0.81-0.95$ & 0.002 & 1.61 & $0.98-2.66$ & 0.06 \\
\hline
\end{tabular}

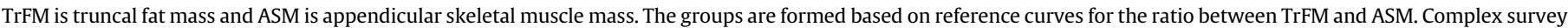
design is taken into account for calculating the TR.

a Accelerated failure time model analysis with lognormal distribution.

b Accelerated failure time model analysis with loglogistic distribution.

c Accelerated failure time model analysis with exponential distribution.

d Adjusted for sex, ethnicity, education level, household income level, smoking status and average daily physical activity.

stratified analysis ( $<70 \mathrm{y}$ and $\geq 70$ years) to evaluate the association between SO and mortality [16]. Furthermore, we used AFT models (with age as time scale and age at baseline survey as entry time) to evaluate the association of SO with survival time since the proportional hazard assumptions were not met. Despite the methodological differences, Batsis et al. [18] also observed a significant increase in mortality risk associated with SO (only in men) and the association was stronger for low lean body mass independent from adiposity. This result could be explained by the age-dependent effect of adiposity on risk of mortality that we observed in our age-stratified analysis.

Other studies did not assess SO using DXA, but they used mid arm circumference [12,17], or muscle function (walking speed [13]; hand grip/knee extensor strength [14-16]) to define sarcopenia. Excess adiposity was defined by anthropometric measurements including BMI $[13,14,16]$ or WC $[12,15,17]$. However, the comparison of results from the various studies is also complicated by the differences in baseline age, sex distribution and duration of follow up; three studies included male participants only with age between 45 and 79 years and follow up range between 6 and 30 years [12,14,17]. Other studies included both middle aged and older men and women with age ranges between 50 and 91 years and follow up duration between 5 and 33 years $[15,16]$ and in one study participants aged between 65 and 102 years which were followed for 6 years [13]. Despite these differences, all these studies reported a significant association of sarcopenia or SO with mortality risk and our results are in line with these studies. Only one study [16] explored this age-interaction and, similar to our results, reported an increased mortality risk in obese and normal-weight participants with low handgrip strength in the 50-69 age group whereas overweight and obese participants aged 70 years and older with high handgrip strength had significantly lower mortality than normal-weight participants. This reversed prediction of adiposity for disease and mortality risk as age increases is a documented observation in epidemiological studies [16,42-45]. The "adiposityage paradox" is based on the notion that excess adiposity is one of the causal steps in the pathogenesis of cardiovascular and metabolic diseases at younger age but this predictive capacity progressively disappears as people age with excess adiposity becoming a protective factor [46].

A strength of this study is the large sample size of 3577 participants and that body composition was analysed with DXA, which is currently the preferred method for body composition assessment based on the accuracy, repeatability and costs. In addition, the new proposed DXA models allowed to control for the confounding effect of age, sex and BMI on the assessment of the body composition phenotypes. In addition, in the survival analysis age was used as time scale instead of time on study. We also excluded individuals who died in the first two years of follow up to minimise the influence of severe illnesses on body composition and mortality at baseline. A limitation of this study is that people taller than $1.96 \mathrm{~m}$ and heavier than $136 \mathrm{~kg}$ were excluded from the analysis, since these people were not eligible for a DXA scan. A consequence of this is that it may not be fully representative of other, more extreme 
body composition phenotypes, such as individuals with morbid obesity. A potential limitation of our approach to identify SO cases is that it is based on the assessment of muscle mass without taking into consideration measures of muscular function as recommended in recent guidelines $[47,48]$. These recommendations follow from findings that the important components of sarcopenia, low skeletal muscle mass and low skeletal muscle function, are not always directly associated and need to be identified separately. Although the assessment of muscular function is key to assess disease and mortality risk, our models aim primarily at improving the sensitivity of DXA-derived measurements of body composition for disease risk prediction. Future studies need to identify to what extent muscle function is able to improve mortality risk prediction based on our body composition models. Our current analysis advanced knowledge in the field by demonstrating a greater sensitivity of the body composition phenotype model and the TrFM/ASM ratio to predict mortality risk and observed that the sensitivity of the models is age-dependent. Future research is warranted to evaluate whether the addition of muscular function to the body composition phenotype and the TrFM/ASM ratio models may improve disease and mortality risk prediction. Another limitation is that body composition was only measured at baseline, and the covariates of the analysis were self-reported. Additionally, the follow-up period was relatively short (7-12 years); however, this was the first study that used the novel DXA models and, even with this relatively short follow-up time, a significant association was found between body composition and mortality risk.

In conclusion, the body composition phenotype model and the TrFM/ASM ratio model are sensitive significant predictors of survival. The preferred model, for future research, should depend on the research question. In addition, SO increases mortality risk in people of 50-70 years, but not in people of 70 years and older. In this group a relatively high FM and high muscle mass seem to be beneficial. More research is needed into the understanding of agerelated differences in the association between body composition and mortality.

\section{Conflict of interest}

The authors have no conflict of interest to declare.

\section{Acknowledgments}

We thank Dr Andrew Kingston for his advisory role in the application of the accelerated time failure models. We would like thank Darren Cole for his help with the development of the automated toolkit. The authors' responsibilities were as follows - MS, CMMP, SH, JCW and PTK: study concept and design; CvA and MS: manuscript drafting; CVA, BCMS and MS data acquisition and analysis; CVA, LMD, CMMP, BCMS, SH, JCW, PTK and MS: and interpretation and critical revision of the manuscript.

\section{Appendix A. Supplementary data}

Supplementary data related to this article can be found at https://doi.org/10.1016/j.clnu.2018.01.022.

\section{References}

[1] Siervo M, Jebb SA. Body composition assessment: theory into practice: introduction of multicompartment models. IEEE Eng Med Biol Mag Q Mag Eng Med Biol Soc 2010;29:48-59.

[2] Hallal PC, Andersen LB, Bull FC, Guthold R, Haskell W, Ekelund U, et al. Global physical activity levels: surveillance progress, pitfalls, and prospects. Lancet 2012;380:247-57.
[3] McAllister EJ, Dhurandhar NV, Keith SW, Aronne LJ, Barger J, Baskin M, et al. Ten putative contributors to the obesity epidemic. Crit Rev Food Sci Nutr 2009;49:868-913.

[4] Chaput J-P, Klingenberg L, Rosenkilde M, Gilbert J-A, Tremblay A, Sjödin A Physical activity plays an important role in body weight regulation. J Obes 2011:360257.

[5] Zamboni M, Zoico E, Scartezzini T, Mazzali G, Tosoni P, Zivelonghi A, et al Body composition changes in stable-weight elderly subjects: the effect of sex. Aging Clin Exp Res 2003;15:321-7.

[6] Baumgartner RN. Body composition in healthy aging. Ann N Y Acad Sc 2000;904:437-48

[7] Prentice AM, Jebb SA. Beyond body mass index. Obes Rev 2001;2:141-7.

[8] Bazzocchi A, Ponti F, Albisinni U, Battista G, Guglielmi G. DXA: technical aspects and application. Eur J Radiol 2016;85:1481-92.

[9] Prado CM, Wells JC, Smith SR, Stephan BC, Siervo M. Sarcopenic obesity: a critical appraisal of the current evidence. Clin Nutr (Edinburgh, Scotland) 2012;31:583-601.

[10] Kohara K. Sarcopenic obesity in aging population: current status and future directions for research. Endocrine 2014;45:15-25.

[11] Donini LM, Poggiogalle E, Migliaccio S, Aversa A, Pinto A. Body composition in sarcopenic obesity: systematic review of the literature. Mediterr J Nutr Metabol 2013;6:191-8.

[12] Atkins JL, Whincup PH, Morris RW, Lennon LT, Papacosta O, Wannamethee SG. Sarcopenic obesity and risk of cardiovascular disease and mortality: a population-based cohort study of older men. J Am Geriatr Soc 2014;62: 253-60.

[13] Cesari M, Pahor M, Lauretani F, Zamboni V, Bandinelli S, Bernabei R, et al. Skeletal muscle and mortality results from the InCHIANTI Study. J Gerontol Ser A Biol Sci Med Sci 2009:gln031.

[14] Rantanen T, Harris T, Leveille SG, Visser M, Foley D, Masaki K, et al. Muscle strength and body mass index as long-term predictors of mortality in initially healthy men. J Gerontol Ser A Biol Sci Med Sci 2000;55:M168-73.

[15] Rossi AP, Fantin F, Caliari C, Zoico E, Mazzali G, Zanardo M, et al. Dynapenic abdominal obesity as predictor of mortality and disability worsening in older adults: a 10-year prospective study. Clin Nutr 2016;35:199-204.

[16] Stenholm S, Mehta NK, Elo IT, Heliövaara M, Koskinen S, Aromaa A. Obesity and muscle strength as long-term determinants of all-cause mortality-a 33 year follow-up of the Mini-Finland Health Examination Survey. Int J Obes 2014;38:1126-32.

[17] Wannamethee SG, Shaper AG, Lennon L, Whincup PH. Decreased muscle mass and increased central adiposity are independently related to mortality in older men. Am J Clin Nutr 2007;86:1339-46.

[18] Batsis JA, Mackenzie TA, Emeny RT, Lopez-Jimenez F, Bartels SJ. Low lean mass with and without obesity, and mortality: results from the 1999-2004 national health and nutrition examination survey. J Gerontol Ser A Biol Sci Med Sci 2017; $72: 1445-51$.

[19] Batsis JA, Mackenzie TA, Barre LK, Lopez-Jimenez F, Bartels SJ. Sarcopenia sarcopenic obesity and mortality in older adults: results from the National Health and Nutrition Examination Survey III. Eur J Clin Nutr 2014;68: $1001-7$.

[20] Hirani V, Naganathan V, Blyth F, Le Couteur DG, Seibel MJ, Waite LM, et al. Longitudinal associations between body composition, sarcopenic obesity and outcomes of frailty, disability, institutionalisation and mortality in community-dwelling older men: the Concord Health and Ageing in Men Project. Age Ageing 2017 May 1:46(3):413-20.

[21] Prado CMM, Siervo M, Mire E, Heymsfield SB, Stephan BCM, Broyles S, et al A population-based approach to define body-composition phenotypes. Am Clin Nutr 2014:99:1369-77.

[22] Siervo M, Prado CM, Mire E, Broyles S, Wells JCK, Heymsfield S, et al. Body composition indices of a load-capacity model: gender-and BMI-specific reference curves. Publ Health Nutr 2015;18:1245-54.

[23] Zipf G, Chiappa M, Porter KS, Ostchega Y, Lewis BG, Dostal J. National health and nutrition examination survey: plan and operations, 1999-2010. Vital Health Stat Ser 1 Prog Coll Procedure 2013:1-37.

[24] Statistics NCfH. Dual energy X-ray absorptiometry (DXA) procedures manual. 2007.

[25] National Center for Health S. National Health and Nutrition Examination Survey: technical documentation for the 1999-2004 dual energy X-ray absorptiometry (DXA) multiple imputation data files. 2008. URL: http://www. cdc.gov/nchs/data/nhanes/dxa/dxa_ techdoc pdf.

[26] George B, Seals S, Aban I. Survival analysis and regression models. J Nucl Cardiol 2014:21:686-94.

[27] Kom EL, Graubard BI, Midthune D. Time-to-event analysis of longitudinal follow-up of a survey: choice of the time-scale. Am J Epidemiol 1997;145: $72-80$.

[28] Heymsfield SB, Gonzalez MC, Lu J, Jia G, Zheng J. Skeletal muscle mass and quality: evolution of modern measurement concepts in the context of sarcopenia. Proc Nutr Soc 2015;74:355-66.

[29] Lee SW, Youm Y, Lee WJ, Choi W, Chu SH, Park Y-R, et al. Appendicular skeletal muscle mass and insulin resistance in an elderly Korean population: the Korean social life, health and aging project-health examination cohort. Diab Metab J 2015;39:37-45.

[30] Janssen I, Baumgartner RN, Ross R, Rosenberg IH, Roubenoff R. Skeletal muscle cutpoints associated with elevated physical disability risk in older men and women. Am J Epidemiol 2004;159:413-21. 
[31] Bosy-Westphal A, Muller MJ. Identification of skeletal muscle mass depletion across age and BMI groups in health and disease-there is need for a unified definition. Int J Obes 2005;39:379-86.

[32] $\mathrm{CDC} / \mathrm{NCHS}$. Deaths, percent of total deaths, and death rates for the 15 leading causes of death in selected age groups, by race and sex: United States. CDC National Center for Health Statistics; 1999-2015.

[33] Bea JW, Thomson CA, Wertheim BC, Nicholas JS, Ernst KC, Hu C, et al. Risk of mortality according to body mass index and body composition among postmenopausal women. Am J Epidemiol 2015;182:585-96.

[34] Chung JH, Hwang HJ, Shin HY, Han CH. Association between sarcopenic obesity and bone mineral density in middle-aged and elderly Korean. Ann Nutr Metabol 2016;68:77-84.

[35] Fabbri E, Chiles Shaffer N, Gonzalez-Freire M, Shardell MD, Zoli M, Studenski SA, et al. Early body composition, but not body mass, is associated with future accelerated decline in muscle quality. J Cachexia Sarcopenia Muscle 2017;8:490-9.

[36] Franca NAG, Peters BSE, Lima MMS, Santos EA, Santos PC, Martini LA. Muscle mass as the main component of body composition associated with bone mineral density. Osteoporos Int 2017;28:S210-1.

[37] Heo M, Faith MS, Pietrobelli A, Heymsfield SB. Percentage of body fat cutoffs by sex, age, and race-ethnicity in the US adult population from NHANES 19992004. Am J Clin Nutr 2012;95:594-602.

[38] Lee S, Kim TN, Kim SH. Sarcopenic obesity is more closely associated with radiographic knee osteoarthritis than non-sarcopenic obesity: a crosssectional study using data from the fifth Korean national health and nutrition examination survey (KNHANES V-1). Ann Rheum Dis Conf Ann Eur Congr Rheumatol Eur Leag Against Rheum EULAR 2012;71.

[39] Li R, Xia J, Zhang X, Gathirua-Mwangi WG, Guo J, Li Y, et al. Associations of muscle mass and strength with all-cause mortality among US older adults. Med Sci Sports Exerc 2018 Mar:50(3):458-67.
[40] Senechal M, Dionne IJ, Brochu M. Dynapenic abdominal obesity and metabolic risk factors in adults 50 years of age and older. J Aging Health 2012;24: 812-26.

[41] Szlejf C, Parra-Rodriguez L, Rosas-Carrasco O. Osteosarcopenic obesity: prevalence and relation with frailty and physical performance in middle-aged and older women. J Am Med Dir Assoc 2017;18:733.e1-5.

[42] Siervo M, Harrison SL, Jagger C, Robinson L, Stephan BC. Metabolic syndrome and longitudinal changes in cognitive function: a systematic review and meta-analysis. J Alzheim Dis JAD 2014;41:151-61.

[43] Osher E, Stern N. Obesity in Elderly Subjects: in sheep's clothing perhaps, but still a wolf! Diabetes Care 2009;32:S398-402.

[44] Lisko I, Tiainen K, Stenholm S, Luukkaala T, Hurme M, Lehtimäki T, et al. Inflammation, adiposity, and mortality in the oldest old. Rejuvenation Res 2012;15:445-52.

[45] Auyeung TW, Lee JSW, Leung J, Kwok T, Leung PC, Woo J. Survival in older men may benefit from being slightly overweight and centrally obese-a 5year follow-up study in 4,000 older adults using DXA. J Gerontol Ser A Biol Sci Med Sci 2010;65A:99-104.

[46] Chapman IM. Obesity paradox during aging. Interdiscipl Top Gerontol 2010;37:20-36

[47] Studenski SA, Peters KW, Alley DE, Cawthon PM, McLean RR, Harris TB, et al. The FNIH sarcopenia project: rationale, study description, conference recommendations, and final estimates. J Gerontol Ser A Biol Sci Med Sci 2014;69: $547-58$.

[48] Cruz-Jentoft AJ, Baeyens JP, Bauer JM, Boirie Y, Cederholm T, Landi F, et al. Sarcopenia: European consensus on definition and diagnosis: report of the European Working Group on sarcopenia in older people. Age Ageing 2010;39: 412-23. 